\title{
Effects of organisational-level interventions at work on employees' health: a systematic review
}

\author{
Diego Montano ${ }^{1 *}$, Hanno Hoven $^{1,2}$ and Johannes Siegrist ${ }^{1}$
}

\begin{abstract}
Background: Organisational-level workplace interventions are thought to produce more sustainable effects on the health of employees than interventions targeting individual behaviours. However, scientific evidence from intervention studies does not fully support this notion. It is therefore important to explore conditions of positive health effects by systematically reviewing available studies. We set out to evaluate the effectiveness of 39 health-related intervention studies targeting a variety of working conditions.
\end{abstract}

Methods: Systematic review. Organisational-level workplace interventions aiming at improving employees' health were identified in electronic databases and manual searches. The appraisal of studies was adapted from the Cochrane Back Review Group guidelines. To improve comparability of the widely varying studies we classified the interventions according to the main approaches towards modifying working conditions. Based on this classification we applied a logistic regression model to estimate significant intervention effects.

Results: 39 intervention studies published between 1993 and 2012 were included. In terms of methodology the majority of interventions were of medium quality, and four studies only had a high level of evidence. About half of the studies (19) reported significant effects. There was a marginally significant probability of reporting effects among interventions targeting several organisational-level modifications simultaneously (Odds ratio (OR) $2.71 ; 95 \% \mathrm{Cl}$ 0.94-11.12), compared to those targeting one dimension only.

Conclusions: Despite the heterogeneity of the 39 organisational-level workplace interventions underlying this review, we were able to compare their effects by applying broad classification categories. Success rates were higher among more comprehensive interventions tackling material, organisational and work-time related conditions simultaneously. To increase the number of successful organisational-level interventions in the future, commonly reported obstacles against the implementation process should be addressed in developing these studies.

Keywords: Occupational health, Employee health, Organisational-level intervention, Effectiveness, Systematic review

\section{Background}

Based on the occupational health principle of "hierarchy of controls" [1] it was proposed that interventions addressing the level of work organisation or work environment may produce more sustainable effects on the health of employees than interventions focusing mainly on individual-level characteristics [2]. This argument is also implicit in influential policy statements and initiatives such as WHO's Global Strategy for Occupational Health for All (1994) [3], the UK Management Standards [4], or the European Directive 89/391 - OSH, among others [5].

\footnotetext{
* Correspondence: diego.montano@med.uni-duesseldorf.de

'Senior Professorship "Work Stress Research", Faculty of Medicine,

Duesseldorf University, Duesseldorf, Germany

Full list of author information is available at the end of the article
}

Thus, workplace interventions improving the working conditions are expected to result in substantial reductions of work-related ill health. Such interventions modify specific circumstances under which work is performed [6]; for instance, exposure to physical and chemical agents, working time and intensity, type of employment contract, psychosocial factors at work, work-life balance, and health and safety policies within the organization. Nevertheless, in contrast to rather consistent findings of several metaanalyses of individual-level interventions reporting statistically significant effects on selected health outcomes (e.g. [7-10]), the results of organisational-level interventions have yielded inconsistent findings so far [11-14]. Several systematic reviews have examined the effects of organisational-

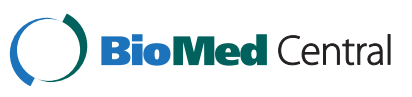


level interventions on specific health-related outcomes such as work-life balance [15], mental health [16], general health and well-being [17], job stress [2], injury prevention [18], and psychosocial and health effects [19]. In these reviews, a lack of consistency of intervention effects was demonstrated and was discussed in methodological and practical terms. For instance, difficulties of engaging employers, but also employees in intervention activities, interference of the intervention with organisational changes and personnel turnover [20], or inability to adjust for a variety of confounding factors were mentioned [21,22]. Moreover, as employees and (line) managers play an active role in determining how and why interventions work [23] there are potential process-related influences which can hardly be controlled [24]. Understanding how employees appraise the intervention itself, how they are involved in the planning, implementation, and process of interventions, and how line managers' behaviours may drive or hinder the intervention process are important factors to be taken into account when designing successful worksite intervention studies $[23,25,26]$.

In methodological terms, the paucity and difficulty of implementing organisational-level interventions with highest degree of evidence, i.e. randomized controlled trials (RCT), is one such limiting factor. Even though some authors claim that RCTs are impractical and inadequate in organisational interventions - arguing that they do not take into account psychological and social processes having a causal relationship with the intervention outcomes [24] -, it should be mentioned that interventions as such can be regarded as the distal causes of organisational change and, thus, should be subject to systematic inquiry in terms of RCTs. Another methodological concern of available intervention studies relates to the high degree of heterogeneity of intervention designs, study protocols, intervention measures and study populations. This heterogeneity prevents rapid progress of cumulative knowledge, and it creates obstacles against systematically reviewing available results.

In this contribution we set out to tackle this latter difficulty by evaluating all organisational-level intervention studies identified by our search strategy on the basis of a systematic classification. This classification aims to synthesise the major modifications of the working conditions implemented by the interventions. We distinguish work organisation-directed changes from work time-directed changes and from changes of the material substrate of work (see Methods). By improving the comparability of the reported study findings we aim at identifying the conditions that may explain the effectiveness of interventions at organisational level. To this end, we apply a logistic regression model to estimate significant intervention effects.

\section{Methods}

Search strategy for the identification of relevant intervention studies

Studies were identified by screening the following databases: ASSIA: Applied Social Sciences Index and Abstracts (SciVerse), Business Source Premier (EBSCO), Cochrane Central Register of Controlled Trials (CENTRAL), Econlit (EBSCO), PubMed (PMC), Scopus (SciVerse), Social Science Citation Index (Web of Knowledge), Sociological Abstracts (ProQuest), and WISO: Wirtschaftswissenschaften. The search was restricted to original papers in peer-reviewed international journals in English, German, French, and Dutch language, published between January 1980 and December 2012. Therefore, neither conference papers or anthologies nor government-commissioned reports were considered. Search terms included various health outcomes, work environment factors, and different intervention methods. The search queries are reported in Additional file 1. The systematic search in databases was amended by search in other systematic reviews, meta-analyses, consulting of experts, and search in relevant websites. Only peer-reviewed studies were assessed. As a quality checklist for reporting the PRISMA statement [27], the Cochrane collaborations tool for risk of bias [28], and the Cochrane Collaboration Back Review Group's 2003 criteria for deciding quality of evidence [29] were adopted.

Two authors (DM, HH) judged all records on the basis of titles and abstracts. In ambiguous cases papers were discussed and full texts were consulted. In a second step, all selected papers were independently reviewed by the two authors based on full texts, and again, ambiguous cases were discussed. Full analysis of the articles and selection of the studies included in the systematic review were made by the first author.

\section{Assessment of the risk of bias and level of evidence}

As stated in the Introduction, the selection of studies in this systematic review was based on two main criteria: (1) organisational-level interventions at the primary prevention level, and (2) studies aiming to improve health-related outcomes. We did not select specific health outcomes, but included all health measures reported in the studies in order to evaluate a relatively large body of evidence. The assessment of risk of bias and level of evidence was based on the method guidelines for systematic reviews proposed by the Cochrane Collaboration $[17,29]$. However, we restricted the quality criteria to factors related to the effectiveness of the intervention (some authors labelled these criteria "internal and statistical validity of a study design" [30]). As can be seen from Table 1, each criterion was given equal weight ( 1 vs. 0 ), except the criterion 'sample size' where we computed quartiles of sample size and assigned weights ranging from 0 (lowest sample sizes) to 3 
Table 1 Quality assessment criteria and weighting scheme

\begin{tabular}{|c|c|c|}
\hline Criteria & Rationale & Weights \\
\hline Is there a control group? & Reduction of bias of the estimates of intervention effects & 0 or 1 \\
\hline Is the baseline response greater than $70 \%$ & Reduction of sampling bias & 0 or 1 \\
\hline Is the follow-up response greater than $50 \%$ & Reduction of attrition and turnover bias & 0 or 1 \\
\hline Have the authors adjusted for non-response and drop-out? & $\begin{array}{l}\text { Reduction of bias of the estimates of intervention effects by } \\
\text { adjusting for attrition and turnover bias }\end{array}$ & 0 or 1 \\
\hline Is there adequate adjustment for the majority of known confounders? & $\begin{array}{l}\text { Reduction of bias of the estimates of intervention effects by } \\
\text { adjusting for relevant confounders }\end{array}$ & 0 or 1 \\
\hline Where appropriate statistical tests used? & $\begin{array}{l}\text { Reduction of bias of the estimates of intervention effects by } \\
\text { using appropriate statistical methods }\end{array}$ & 0 or 1 \\
\hline $\begin{array}{l}\text { Sample size at baseline. } 0 \text { if } n \text { is in 1st quantile, } 2 \text { if } n \text { is in the } \\
2 n d \text { quantile, } 3 \text { if } n \text { is in the } 3 r d \text { quantile }\end{array}$ & Validity of statistical inference & 0 to 3 \\
\hline $\begin{array}{l}\text { Sample size at follow-up. } 0 \text { if } n \text { is in 1st quantile, } 2 \text { if } n \text { is in the } \\
2 n d \text { quantile, } 3 \text { if } n \text { is in the } 3 r d \text { quantile. }\end{array}$ & Validity of statistical inference & 0 to 3 \\
\hline
\end{tabular}

(highest sample sizes). The reason of emphasizing sample size within the evaluation of study quality is the lack of information on statistical power within the reviewed studies. We therefore used sample size at baseline and at follow-up as proxies of potential statistical power estimates of meeting significant intervention effects. The total quality of each study was defined as the sum of scores obtained in each quality criterion, varying from 0 (lowest) to 12 (highest).

As an additional relevant criterion of evaluation, the level of evidence was assessed by taking into account the study design (Table 2). By level of evidence we mean the extent to which a study is capable of detecting and estimating a real intervention effect with minimum bias. In order to simplify the presentation of results, three levels of evidence only were defined. The highest level of evidence in the context of our systematic review was assumed for randomized controlled trials (RCT) whose quality score as defined before - had to meet a level above the median of the quality scores of all studies. Given the fact that randomisation minimises the risk of bias in comparison with all other designs [31], this type of study design is likely to produce the relatively strongest degree of evidence of an intervention effect [32]. However, given the difficulties of RCTs discussed above, quasi-experimental designs are generally applied more often in this field of research. If they contain well-defined control groups [33], quasiexperimental studies meeting the defined quality standard are judged as meeting a medium level of evidence. They share this level with those RCT studies that failed to reach the defined quality standard. All remaining studies were classified as belonging to a low category of evidence, being vulnerable to confounding bias [30,34].

\section{Classification of the major modifications of the working conditions implemented by the interventions}

Given the complexity and variety of implementations in the interventions we proposed three broad categories, referring to a classification of conditions of work used in the European Working Conditions Survey [35,36].

1. Material conditions. All sorts of physical and chemical agents implied in the execution of work tasks are included (e.g. vibrations, noise, chemical substances, ergonomics).

2. Work time-related conditions. These conditions relate to the amount of working time and intensity of work, the latter being measured as number of activities per time unit (e.g. work speed, shifts, deadlines, pace of work, breaks).

3. Work organisation conditions. These include a variety of psychological and social factors (job demands, job control, efforts and rewards, responsibility, etc.), and processes and procedures required to accomplish work tasks (e.g. methods of work, order of tasks, team organization, structure of hierarchy, security guidelines training).

Table 2 Scheme of level of evidence for each single study

\begin{tabular}{ll}
\hline Level & Definition \\
\hline High & The study has a randomized controlled design whose quality is greater than the median study quality. \\
Medium & The study is either a randomized controlled design whose quality is less than the median study quality, \\
& or the study is a quasi-experimental control study whose quality is greater than the median study quality \\
Low & The study is either a quasi-experimental control trial whose quality is less than the median study quality, \\
& or the study is a quasi-experimental one-group prospective or a cross-sectional one.
\end{tabular}


This classification is not exclusive as studies may address more than one intervention target simultaneously. In fact, it is our aim to explore to what extent a more comprehensive intervention is better suited to produce significant health effects, compared to interventions that are restricted to one single target (see below). We exemplify our classification procedure by illustrating one case in more detail [37]. The purpose of the intervention of Morken and colleagues was to enable operators and the organisation to prevent and cope with musculoskeletal problems at the workplace. The intervention consisted of ten didactic sessions during which operators found solutions on how to obtain an optimal work environment both organisationally and technically. Some of the changes implemented during the intervention period were redesigning the workplace, reducing repetitive-motion stress points and modifying the work process to promote job variations. According to our classification scheme, these changes can be classified as changes of the material and organisational conditions of work since they include both ergonomic and work process changes. It should be noted that by roughly merging our classification of intervention targets with the classification of the European Working Conditions Survey, we intend to advance the comparison of results of intervention studies with results of surveys, thus strengthening cross-fertilization and policy impact of the two research strands (see e.g. [38]).

\section{Estimation of the probability of reporting significant intervention effects}

In order to evaluate to what extent modification of the working conditions may be associated with the reporting of statistically significant effects we estimated a logistic regression model. To this end, we define the reporting of significant intervention effects (which we consider a proxy of intervention effectiveness) as dependent variable. Clearly, the heterogeneity of study designs and of outcome variables does not allow a meta-analysis. We therefore restrict our quantitative analysis to the test of a research hypothesis. Our hypothesis claims that the probability of reporting statistically significant effects increases with an increased degree of comprehensiveness of intervention targets. To test this hypothesis a logistic regression model was used to estimate the probability of reporting positive intervention effects as a function of the number of working conditions changed by the intervention.

There are at least two major confounders which have to be addressed when testing the hypothesis. First, it may be that studies addressing several intervention targets simultaneously score higher in terms of study quality, including study design. Therefore, we control for this effect by appropriate adjustment in the regression model. Second, since the socioeconomic characteristics of the samples define a particular work environment and social setting that usually influences the intervention effectiveness $[39,40]$, we control for this effect by classifying the study samples according to an internationally established classification of occupational positions, the Erikson-Goldthorp-Portocarrero scheme (EGP) [41]. Due to the lack of specific information regarding the occupations of the employees in some of the studies, two major groups of occupations only could be broadly identified:

1. EGP classes I to III. Higher and lower service occupations, routine clerical and sales occupations (e.g. managers, professionals, routine clerical and sales workers).

2. EGP classes VI to VII. Skilled manual and semi- or unskilled manual occupations (e.g. craft workers, skilled service, skilled machine operations, and elementary labourers).

As logistic regression models overestimate the odds ratios in small sample sizes [42], odds ratios were estimated by penalized maximum likelihood and hypothesis testing by likelihood-ratio tests $[43,44]$. All analyses were conducted with the programming language $\mathrm{R}$.

\section{Results}

The search in the databases and the manual search resulted in 18145 initial records after checking for duplicates. Based on titles and abstracts 699 studies were submitted to further appraisal. Applying the selection criteria mentioned in the Methods Section 77 studies were selected for detailed analysis. Of these, 39 intervention studies meeting fully the criteria were included in the systematic review. A detailed description of main characteristics of the studies and their implementation is reproduced in Additional file 2: Tables $\mathrm{S} 1$ and S2.

A brief overview of the main characteristics of the studies is reproduced in Table 3. The majority of studies followed a participatory approach, and many investigations applied a quasi-experimental prospective design with a control group. The median of follow-up time was one year. About half of the studies reported statistically significant intervention effects on health-related outcomes. Four studies were considered as having a high level of evidence. In a majority of studies employees belonged to occupational classes I to III. Only six studies included all occupational classes. Studies were conducted mostly in urban areas and targeted usually health care workers (at least 14 studies), manufacture workers (at least four studies) and civil servants (at least three studies). Sample sizes at baseline varied considerably in the range of 41 to 3506 participants, even though 28 out of 39 studies had sample sizes less than 500 employees.

An analysis of the frequencies of specific outcomes showed that interventions focused mostly on burnout 
Table 3 Descriptive statistics of studies reviewed

\begin{tabular}{|c|c|}
\hline Variable & $\begin{array}{c}\text { Number of studies/ } \\
\text { median and range }\end{array}$ \\
\hline \multicolumn{2}{|l|}{ EGP occupational class } \\
\hline$|-|||$ & 18 \\
\hline$|-| I I, V I-V I I$ & 6 \\
\hline $\mathrm{VI}-\mathrm{VII}$ & 15 \\
\hline \multicolumn{2}{|l|}{ Study design } \\
\hline Quasi-experimental, cross-sectional & 1 \\
\hline Quasi-experimental one-group prospective & 9 \\
\hline $\begin{array}{l}\text { Quasi-experimental prospective with } \\
\text { control group }\end{array}$ & 21 \\
\hline $\begin{array}{l}\text { Quasi-experimental prospective } \\
\text { and retrospective }\end{array}$ & 2 \\
\hline Randomized controlled & 6 \\
\hline \multicolumn{2}{|l|}{ Evidence grade } \\
\hline High & 4 \\
\hline Medium & 25 \\
\hline Low & 10 \\
\hline \multicolumn{2}{|l|}{ Significant intervention effects } \\
\hline No & 21 \\
\hline Yes & 18 \\
\hline \multicolumn{2}{|l|}{ Intervention type } \\
\hline Organisational intervention (OI) & 9 \\
\hline $\begin{array}{l}\text { Participatory (including Participatory } \\
\text { Ergonomics PE and Participatory } \\
\text { Research Action PAR) }\end{array}$ & 7 \\
\hline Shift schedules & 18 \\
\hline Other & 5 \\
\hline Year of publication & $2005[1993,2012]$ \\
\hline Sample size at baseline & $300[41,3506]$ \\
\hline Sample size at follow-up & $187[36,2617]$ \\
\hline Sample size of control group & $122[31,347]$ \\
\hline Follow-up time (years) & $1[0.25,13]$ \\
\hline Study quality & $7[1,11]$ \\
\hline Number of working conditions changed & $2[1,4]$ \\
\hline
\end{tabular}

(six studies), absenteeism (four studies), musculoskeletal disorders of the upper body (six studies), and depressive symptoms (three studies). The studies were conducted in the following industrialized countries: Australia, Canada, Italy, Germany, the Scandinavian countries, USA, Japan, UK and The Netherlands.

In Table 4 the frequencies of reporting statistically significant effects for each category of working conditions are reported. Ten studies introduced changes in the material and organisational conditions, 16 studies concentrated on organisational conditions, whereas eight studies emphasised on the time conditions of work. Three studies comprised all three types of working conditions and reported
Table 4 Types of modified working conditions and frequency of significant intervention effects

\begin{tabular}{lccc}
\hline Working condition & $\begin{array}{c}\text { Number of } \\
\text { modified working } \\
\text { conditions }\end{array}$ & \multicolumn{2}{c}{$\begin{array}{c}\text { Statistically } \\
\text { significant? }\end{array}$} \\
\cline { 2 - 4 } & 1 & 1 & Yes \\
\hline Material & 2 & 4 & 6 \\
Material, organisation & 3 & 0 & 3 \\
Material, time, organisation & 1 & 10 & 6 \\
Organisation & 1 & 3 & 2 \\
Time & 2 & 2 & 1 \\
Time, organisation & & & 6
\end{tabular}

statistically significant effects on burnout and injury prevention [45-47]. The changes implemented by these interventions included staffing processes, work reorganisation, training, ergonomics, assessment of factors relating to iterative injuries, and control processes.

At the same time, interventions modifying material and organisational conditions of work also report more frequently significant effects (six out of ten studies). These interventions involved, among others, the introduction of mechanical lifting equipment and employee training [48], improvements of machine performance and communication between workers and supervisors [49], reduction of lifting loads and rotation schedules [50], increased compliance with the use of protective substances and establishment of improved control procedures of occupational risks [51], improvement of technical equipment and health surveillance [52], as well as substituting chemicals and increased safety guidelines in the organisation [53]. The health-related outcomes of these interventions included back pain, injuries, sick leave, blood pressure, and eczema.

If the results of each intervention study are compared according to their level of evidence, three out of four high-evidence studies did not report significant intervention effects $[37,54,55]$, whereas 14 out of 25 medium evidence level studies reported significant improvements of health-related outcomes, such as ischemic heart disease risk [56], burnout $[45,46,57,58]$, lost time injury [47], perceived health [59], blood pressure [60], decreased mental distress and better sleep [61], reduction of sick-leave length [49], back-pain related lost working days [50], eczema incidence [53], and mental health [62]. Finally, four out of ten low evidence studies reported statistically significant changes in outcomes such as self-rated health [63] and injury rates $[48,52,64]$.

Concerning our hypothesis of an association between the probability of reporting statistically significant effects and the number of modified working conditions, results of a respective logistic regression model are given in Table 5 . This analysis takes into account the potential impact of 
Table 5 Logistic regression model estimated by penalized maximum likelihood $^{\mathrm{a}}$

\begin{tabular}{lcccc}
\hline & OR & Lower 95\% Cl & Upper 95\% Cl & P \\
\hline $\begin{array}{l}\text { Number of modified } \\
\text { Working conditions }\end{array}$ & 2.71 & 0.94 & 11.12 & 0.07 \\
$\begin{array}{l}\text { Study quality } \\
\text { EGP class }\end{array}$ & 0.95 & 0.75 & 1.18 & 0.63 \\
Reference I-III & & & & \\
I-III, VI-VII & 0.42 & 0.05 & 2.66 & 0.37 \\
VI-VII & 0.73 & 0.14 & 3.14 & 0.68 \\
\hline
\end{tabular}

${ }^{a}$ Dependent variable: reporting of significant effects (coded yes/no). Working conditions and study quality treated as metric variables. Pseudo $\mathrm{R}^{2}=0.12$.

occupational class of employees as well as the quality of the study. As can be seen, the number of working conditions modified by the intervention tends to be associated with increased intervention success (even though only marginally significant, $\mathrm{OR}=2.71,95 \% \mathrm{CI} 0.94-11.12$ ). In line with the findings given in Table 4, it seems that interventions addressing different types of working conditions simultaneously may increase the chance of producing a beneficial effect on the outcomes under study. When interpreting the result, it should be kept in mind that two of the three papers analysing comprehensive interventions result from the same intervention study, exploring health outcomes after one year [45] and after three years [46].

\section{Discussion}

In this systematic review 39 organisational-level workplace interventions were analysed with regard to their effects on employee health. We tackled the large heterogeneity of study designs, intervention targets and health outcomes by classifying the studies according to three general categories of intervention targets (material, organisational, work timerelated). About half of the studies yielded statistically significant intervention effects. Favourable health outcomes were reported for self-rated mental and general health, and for reduction of injury rates. Taking the potential impact of study quality and occupational class into account, our main finding revealed that more comprehensive interventions addressing several organisational-level targets simultaneously had a higher chance of reporting significant health improvements than those restricted to one intervention target. Before discussing limitations and strengths of this study the following questions are addressed. First, how can we explain the relatively low success rate of these interventions? And second, how can we interpret the finding that more comprehensive interventions are more likely to result in positive health outcomes among employees?

The success rate of the 39 interventions reviewed in this study is substantially lower than the one reported in an important earlier systematic review by LaMontagne et al. [2] where about two thirds of the 18 intervention studies demonstrated a significant effect. It seems particularly worrying that three of the four studies with highest level of evidence in our review failed to achieve a positive outcome $[37,54,55])$. Therefore, it is instructive to learn how the authors themselves explained the limited success of their interventions. In a synthesis of their arguments the following reasons can be distinguished. First, in some cases there was not sufficient participation of employees in preparing the intervention, or there was a lack of communication and motivation to support the intervention and to comply with organisational changes [65-68]. Second, the implementation of some interventions was difficult because some aspects did not work as anticipated or could not be realised as originally designed $[39,55]$.Third, there were difficulties in developing or maintaining the intervention due to lack of support from employers and managers $[62,66,69]$. Fourth, the intervention was threatened by external events or conditions that were beyond the control of the responsible team (e.g. job turnover, organisational restructuring or merging) [52,53,70]. Finally, the lack of significant effects was eventually due to a short follow-up time, to a weak treatment effect, or to a failure of controlling for potential confounders of intervention effects $[21,58,65,68,69,71]$. These reasons clearly point to the need of developing interventions in a participatory approach, where "organizational interventions (are) to be understood as a collective of initiatives and change activities, competing and intertwining with a multitude of concurrent events" [72]. Moreover, careful process evaluation should be a mandatory part of any intervention trial.

How can we interpret the finding that more comprehensive interventions are more likely to result in positive health outcomes among employees? It is of interest to note that individual-level stress management interventions demonstrated exactly the opposite trend. Effect sizes were strongest if the treatment focused on cognitive behavioural therapy or on an intervention that strengthened employees' resources of coping with stressful work, whereas the effects of multimodal interventions were much weaker [9]. In case of organisational-level interventions, the situation is different. Given the many difficulties of implementation, including the interference of a structural change with established organisational procedures, it may be crucial to develop a comprehensive set of modifications that can exert sufficient impact on employees who are exposed to a complex work environment. This is, for instance, the case if material improvements of work tasks are linked with changes in the division of work or other features of work organisation, and/or with increased flexibility of work schedules. Thus, the combination of a hierarchy of controls approach with specific improvements of technical, chemical or physical elements of the work environment may contribute to a reduction of risk exposure and related adverse health effects. 


\section{Limitations}

This systematic review has several limitations. First, the inconsistency of health effects of interventions regarding changes of the working conditions should be interpreted with caution, since study designs, outcomes and corresponding measures are highly heterogeneous. Second, it is possible that we bypassed some relevant intervention studies during our search strategy, in particular as we did not include intervention studies published in books, anthologies, working papers or other grey literature sources. Third, neither the weighting scheme of the study quality nor the classification of working conditions is based on internationally adopted procedures. The broad classification of working conditions may obscure potential significant effects of specific intervention designs within each category. For instance, if we had classified all studies based on a distinct intervention design (e.g. job-demand-control model [73]) separately, i.e. independent of the three intervention approaches underlying our systematic review, different frequencies of successful interventions could have resulted. As an alternative classification, the presence or absence of an explicit theoretical basis of intervention could be applied, especially so in the area of a healthadverse psychosocial work environment. For instance, many respective interventions were based on the jobdemand-control model. Yet, success rates were not significantly higher, probably due to the difficulties of implementation discussed above. Fourth, the scope of occupations exposed to organisational-level interventions was limited, and a substantial part of the studies under review was addressed to health care workers (15 studies or about $38 \%$ ), thus limiting the generalisation of results. Finally, it cannot be ruled out that some intervention effects are actually due to regression to the mean which may overestimate the intervention effects.

These limitations are balanced by several strengths. First, we collected a relatively large number of intervention studies from different countries, addressing modifications at the organisational level to different occupational groups and exploring different health outcomes. By doing so we minimised a selection bias of results answering our main research question. Second, we tackled the challenge of diversity of study designs and intervention targets by classifying the studies into categories amenable to comparative analysis. Classification schemes concerned the quality of studies and the type of organisational-level interventions. Third, this procedure enabled us to estimate which factors were likely to produce favourable health outcomes among employees. More specifically, we tested the hypothesis that this was the case if interventions were designed as comprehensive modifications, combining material, organisational, and work time-related improvements.

\section{Conclusions}

Despite the heterogeneity of the 39 organisational-level workplace interventions underlying this review, we were able to compare their effects by applying broad classification categories. About half of the studies reveal significant effects on employees' health. Success rates were higher among more comprehensive interventions tackling material, organisational and work-time related conditions simultaneously. To increase the number of successful organisational-level interventions in the future commonly reported obstacles against the implementation process should be addressed in developing these studies.

\section{Additional files}

Additional file 1: Search Queries.

Additional file 2: Results Tables.

\section{Competing interests}

The authors declare that they have no competing interests.

\section{Authors' contributions}

DM contributed substantially to the search strategy, study appraisal, statistical analyses and manuscript preparation. $\mathrm{HH}$ contributed to the development of the search strategy and collaborated in performing the study appraisal and manuscript preparation. JS led the drafting of the manuscript and prepared the final version. All authors read and approved the final manuscript.

\section{Acknowledgements}

The research leading to these results was done within the framework of the DRIVERS project (www.health-gradient.eu) coordinated by EuroHealthNet, and has received funding from the European Community (FP7 2007-2013) under grant agreement no 278350. Johannes Siegrist and Diego Montano were additionally supported by a Senior Professorship Grant from the Faculty of Medicine, University of Duesseldorf, Germany.

\section{Author details}

'Senior Professorship "Work Stress Research", Faculty of Medicine, Duesseldorf University, Duesseldorf, Germany. ${ }^{2}$ Institute of Medical Sociology, Faculty of Medicine, Duesseldorf University, Duesseldorf, Germany.

Received: 28 August 2013 Accepted: 6 February 2014

Published: 8 February 2014

\section{References}

1. Halperin WE: The role of surveillance in the hierarchy of prevention. Am J Ind Med 1996, 29:321-323.

2. LaMontagne AD, Keegel T, Louie A, Ostry A, Landsbergis P: A systematic review of the job-stress intervention evaluation literature, 1990-2005. Int J Occup Environ Health 2007, 13:268-280.

3. WHO: Global strategy on occupational health for All: the way to health at work. Geneva: World Health Organization; 1995.

4. Health and Safety Executive: Managing the causes of work-related stress: A step-by-step approach using the management standards. 2nd edition. Great Britain: HSE; 2007.

5. Finnish Institute of Occupational Health: Well-Being at Work: New Innovations and Good Practices. Helsinki: Finnish Institute of Occupational Health; 2008.

6. Parent-Thirion A: 5th European working conditions survey: overview report. Luxembourg: Publ. Off. of the Europ. Union; 2012.

7. Conn VS, Hafdahl AR, Cooper PS, Brown LM, Lusk SL: Meta-analysis of workplace physical activity interventions. Am J Prev Med 2009, 37:330-339.

8. Martin A, Sanderson K, Cocker F: Meta-analysis of the effects of health promotion intervention in the workplace on depression and anxiety symptoms. Scand J Work Environ Health 2009, 35:7-18. 
9. Richardson KM, Rothstein HR: Effects of occupational stress management intervention programs: a meta-analysis. J Occup Health Psychol 2008, 13:69-93.

10. Fitzpatrick $\mathrm{S}$, Johnsen $\mathrm{S}$, White M: Multiple exclusions homelessness in the UK: key patterns and intersections. Soc Policy Society 2011, 10:501-512.

11. Briner RB, Reynolds S: The costs, benefits, and limitations of organizational level stress interventions. J Organ Behav 1999, 20:647.

12. Nielsen ML, Kristensen TS, Smith-hansen L: The intervention project on absence and well-being (IPAW): design and results from the baseline of a 5-year study. Work \& Stress 2002, 16:191-206.

13. Bambra C, Gibson M, Sowden A, Wright K, Whitehead M, Petticrew M: Working for health? Evidence from systematic reviews on the effects on health and health inequalities of organisational changes to the psychosocial work environment. Prev Med 2009, 48:454-461.

14. Taris1 TW, Kompier1 MA, Geurts SAE1, Schreurs PJG2, Schaufeli WB2, de Boer E3, Sepmeijer KJ3, Wattez C3: Stress management interventions in the Dutch domiciliary care sector: findings from 81 organizations. [Article]. Int J Stress Manage 2003, 10:297-325.

15. Brough $P, O^{\prime}$ Driscoll MP: Organizational interventions for balancing work and home demands: an overview. Work \& Stress 2010, 24:280-297.

16. Corbiere M, Shen J, Rouleau M, Dewa CS: A systematic review of preventive interventions regarding mental health issues in organizations. Work 2009, 33:81-116.

17. Joyce K, Pabayo R, Critchley JA, Bambra C: Flexible working conditions and their effects on employee health and wellbeing. Cochrane Database Syst Rev 2010, 17(2):CD008009.

18. van der Molen HF, Lehtola MM, Lappalainen J, Hoonakker PL, Hsiao H, Haslam $\mathrm{R}$, Hale AR, Frings-Dresen MH, Verbeek JH: Interventions to prevent injuries in construction workers. Cochrane Database Syst Rev 2012, 12, CD006251.

19. Bambra C, Egan M, Thomas S, Petticrew M, Whitehead M: The psychosocial and health effects of workplace reorganisation. 2. A systematic review of task restructuring interventions. J Epidemiol Community Health 2007, 61:1028-1037

20. Olsen O, Albertsen K, Nielsen M, Poulsen K, Gron S, Brunnberg H: Workplace restructurings in intervention studies - a challenge for design, analysis and interpretation. BMC Med Res Methodol 2008, 8:39.

21. Nielsen K, Taris TW, Cox T: The future of organizational interventions: addressing the challenges of today's organizations. Work \& Stress 2010 24:219-233

22. Egan M, Bambra C, Petticrew M, Whitehead M: Reviewing evidence on complex social interventions: appraising implementation in systematic reviews of the health effects of organisational-level workplace interventions. J Epidemiol Community Health 2009, 63:4-11.

23. Nielsen K: Review article: how can we make organizational interventions work? Employees and line managers as actively crafting interventions. Human Relat 2013, 66:1029-1050.

24. Nielsen K, Randall R: Opening the black box: presenting a model for evaluating organizational-level interventions. Eur J Work Organ Psy 2012, 22:601-617.

25. Nielsen K, Randall R: The importance of employee participation and perceptions of changes in procedures in a teamworking intervention. Work \& Stress 2012, 26:91-111.

26. Nielsen K, Randall R, Albertsen K: Participants' appraisals of process issues and the effects of stress management interventions. J Organ Behav 2007, 28:793-810.

27. Moher D, Liberati A, Tetzlaff J, Altman DG: Preferred reporting items for systematic reviews and meta-analyses: the PRISMA statement. PLoS Med 2009, 6:e1000097.

28. Higgins JPT, Green S (Eds): Cochrane Handbook for Systematic Reviews of Interventions: Version 5.1.0 [updated March 2011]; 2011. http://www.cochrane-handbook.org

29. van Tulder M, Furlan A, Bombardier C, Bouter L: Updated method guidelines for systematic reviews in the cochrane collaboration back review group. Spine 2003, 28:1290-1299.

30. Cook TD, Campbell DT: Quasi-experimentation: Design \& analysis issues for field settings. Boston: Houghton Mifflin; 1979.

31. Neyman J, Dabrowska DM, Speed TP: On the application of probability theory to agricultural experiments. Essay on principles. Section 9. Stat Sci 1990, 5:465-472.

32. Barton S: Which clinical studies provide the best evidence? The best RCT still trumps the best observational study. BMJ 2000, 321:255-256.
33. Grant AM, Wall TD: The Neglected science and art of quasi-experimentation: why-to, when-to, and how-to advice for organizational researchers. Organ Res Methods 2009, 12:653-686.

34. Thyer B: Quasi-experimental research designs. New York: Oxford Univ. Press; 2012

35. Eurostat: Labour market statistics. Luxembourg: Publications Office of the European Union; 2011

36. Morley J (Ed): Fourth European Working Conditions Survey: Contribution to policy development. Dublin: European Foundation for the Improvement of Living and Working Conditions; 2002.

37. Morken T, Moen B, Riise T, Hauge SH, Holien S, Langedrag A, Olson $\mathrm{H}_{4}$ Pedersen S, Saue IL, Seljebo GM, Thoppil V: Effects of a training program to improve musculoskeletal health among industrial workers - Effects of supervisors role in the intervention. Int J Ind Ergonom 2002, 30:115-127.

38. Jettinghof K, Houtman I: Sectoral profiles of working conditions. Dublin: European Foundation for the Improvement of Living and Working Conditions; 2009.

39. Saksvik $P \varnothing$, Nytrø K, Dahl-Jørgensen C, Mikkelsen A: A process evaluation of individual and organizational occupational stress and health interventions. Work \& Stress 2002, 16:37-57.

40. van der Molen HF, Sluiter JK, Hulshof CTJ, Vink P, van Duivenbooden C, Frings-Dresen MHW: Conceptual framework for the implementation of interventions in the construction industry. Scand J Work Environ Health 2005, 31(Suppl 2):96-103.

41. Ganzeboom HBG, Treiman DJ: Internationally comparable measures of occupational status for the, international standard classification of occupations. Soc Sci Res 1988, 1996(25):201-239.

42. Nemes S, Jonasson JM, Genell A, Steineck G: Bias in odds ratios by logistic regression modelling and sample size. BMC Med Res Methodol 2009, 9:56.

43. Firth D: Bias reduction of maximum likelihood estimates. Biometrika 1993, $80: 27-38$

44. Bull SB, Mak C, Greenwood CM: A modified score function estimator for multinomial logistic regression in small samples. Comput Stat Data Anal 2002, 39:57-74.

45. Bourbonnais R, Brisson C, Vinet A, Vezina M, Abdous B, Gaudet M: Effectiveness of a participative intervention on psychosocial work factors to prevent mental health problems in a hospital setting. Occup Environ Med 2006, 63:335-342.

46. Bourbonnais $\mathrm{R}$, Brisson C, Vézina M: Long-term effects of an intervention on psychosocial work factors among healthcare professionals in a hospital setting. Occup Environ Med 2011, 68:479-486.

47. Carrivick PJW, Lee AH, Yau KKW: Effectiveness of a workplace risk assessment team in reducing the rate, cost, and duration of occupational injury. J Occup Environ Med 2002, 44:155-159.

48. Collins JW, Wolf L, Bell J, Evanoff B: An evaluation of a "best practices" musculoskeletal injury prevention program in nursing homes. Inj Prev 2004, 10:206-211.

49. Kawakami N, Araki S, Kawashima M, Masumoto T, Hayashi T: Effects of work-related stress reduction on depressive symptoms among Japanese blue-collar workers. Scand J Work Environ Health 1997, 23:54-59.

50. Lemstra M, Olszynski WP: The effectiveness of standard care, early intervention, and occupational management in worker's compensation claims. Spine 2003, 28:299-304.

51. Mygind K, Borg V, Flywholm M, Sell L, Jepsen KF: A study of the implementation process of an intervention to prevent work-related skin problems in wet-work occupations. Int Arch Occup Environ Health 2006, 79:66-74.

52. Porru S, Calza S, Arici C: An effectiveness evaluation of a multifaceted preventive intervention on occupational injuries in foundries: a 13-year follow-up study with interrupted time series analysis. Int Arch Occup Environ Health 2011, 84:867-876.

53. Rasmussen K, Glasscock DJ, Hansen ON, Carstensen O, Jepsen JF, Nielsen KJ: Worker participation in change processes in a Danish industrial setting. Am J Ind Med 2006, 49:767-779.

54. Oude Hengel KM, Blatter BM, Joling Cl, van der Beek AJ, Bongers PM: Effectiveness of an intervention at construction worksites on work engagement, social support, physical workload, and need for recovery: results from a cluster randomized controlled trial. BMC Public Health 2012, 12:1008.

55. Schrijnemaekers VJJ, van Rossum E, Candel MJJM, Frederiks CMA, Derix MMA, Sielhorst $H$, van den Brandt PA: Effects of emotion-oriented care on work-related outcomes of professional caregivers in homes for elderly persons. J Gerontol B-Psychol 2003, 58:S50. 
56. Boggild $H$, Jeppesen $H J$ : Intervention in shift scheduling and changes in biomarkers of heart disease in hospital wards. Scand J Work Environ Health 2001, 27:87-96.

57. Mikkelsen A, Saksvik $P \varnothing$, Landsbergis P: The impact of a participatory organizational intervention on job stress in community health care institutions. Work Stress 2000, 14:156-170.

58. Nabe-Nielsen K, Garde AH, Diderichsen F: The effect of work-time influence on health and well-being: a quasi-experimental intervention study among eldercare workers. Int Arch Occup Environ Health 2011, 84:683-695.

59. DeJoy DM, Wilson MG, Vandenberg RJ, McGrath-Higgins AL, Griffin-Blake CS: Assessing the impact of healthy work organization intervention. J Occup Organ Psych 2010, 83:139-165.

60. Evans GW, Johansson G, Rydstedt L: Hassles on the job: a study of a job intervention with urban bus drivers. J Organ Behav 1999, 20:199.

61. Garde AH, Nabe-Nielsen K, Aust B: Influence on working hours among shift workers and effects on sleep quality - an intervention study. Appl Ergon 2011, 42:238-243.

62. Tsutsumi A, Nagami M, Yoshikawa T, Kogi K, Kawakami N: Participatory intervention for workplace improvements on mental health and job performance among blue-collar workers: a cluster randomized controlled trial. J Occup Environ Med 2009, 51:554-563.

63. Anderzén I, Arnetz BB: The impact of a prospective survey-based workplace intervention program on employee health, biologic stress markers, and organizational productivity. J Occup Environ Med 2005, 47:671-682.

64. Evanoff BA, Bohr PC, Wolf LD: Effects of a participatory ergonomics team among hospital orderlies. Am J Ind Med 1999, 35:358-365.

65. Melchior ME, Philipsen H, Abu-Saad HH, Halfens RJ, van de Berg AA, Gassman P: The effectiveness of primary nursing on burnout among psychiatric nurses in long-stay settings. J Adv Nurs 1996, 24:694-702.

66. Mattila P, Elo A, Kuosma E, Kylä-Setälä E: Effect of a participative work conference on psychosocial work environment and well-being. Eur J Work Organ Psy 2006, 15:459-476.

67. Andersen I, Borritz M, Christensen KB, Diderichsen F: Changing job-related burnout after intervention-a quasi-experimental study in six human service organizations. J Occup Environ Med 2010, 52:318-323.

68. Dahl-Jorgensen C, Saksvik PO: The impact of two organizational interventions on the health of service sector workers. Int J Health Serv 2005, 35:529-549.

69. Logan MS, Ganster DC: An experimental evaluation of a control intervention to alleviate job-related stress. J Manage 2005, 31:90-107.

70. Petterson I, Arnetz BB: Psychosocial stressors and well-being in health care workers. The impact of an intervention program. Soc Sci Med 1998, 47:1763-1772.

71. Garde AH, Albertsen K, Nabe-Nielsen K, Carneiro IG, Skotte J, Hansen SM, Lund $H, H$ vid $H$, Hansen AM: Implementation of self-rostering (the PRIO project): effects on working hours, recovery, and health. Scand J Work Environ Health 2012, 38:314-326.

72. Nielsen K, Abildgaard JS: Organizational interventions: a research-based framework for the evaluation of both process and effects. Work \& Stress 2013, 27:278-297.

73. Karasek R, Theorell T: Healthy work: stress, productivity, and the reconstruction of working life. New York, NY: Basic Books; 1990.

\section{doi:10.1186/1471-2458-14-135}

Cite this article as: Montano et al:: Effects of organisational-level interventions at work on employees' health: a systematic review. BMC Public Health 2014 14:135.

\section{Submit your next manuscript to BioMed Central and take full advantage of:}

- Convenient online submission

- Thorough peer review

- No space constraints or color figure charges

- Immediate publication on acceptance

- Inclusion in PubMed, CAS, Scopus and Google Scholar

- Research which is freely available for redistribution 